\title{
Análisis bibliográfico sobre la brecha digital y la alfabetización en nuevas tecnologías
}

\author{
Bibliographic Analysis of the Digital Divide and Literacy in New Technologies

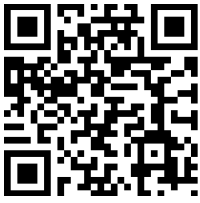 \\ Jesús De Benito-Castanedo ${ }^{1}$ \\ Santander (Cantabria), España \\ jdbcastanedo@hotmail.com \\ http://orcid.org/0000-0003-1476-0441
}

Recibido 5 de febrero de 2016 • Corregido 20 de marzo de 2017 • Aceptado 15 de abril de 2017

\begin{abstract}
Resumen: El artículo analiza el fenómeno de la brecha digital en España, desde una perspectiva bibliográfica, centrándose en la alfabetización digital como elemento pedagógico fundamental. Las intervenciones educativas en materia de nuevas tecnologías han sido objeto de análisis, tanto a nivel académico como institucional, de los cuales se han desprendido múltiples ideas y estrategias para llevar a cabo proyectos de alfabetización digital. Así, el presente escrito refleja la conceptualización y estado actual de la brecha digital, para lo cual considera las intervenciones en alfabetización digital como elemento clave en el proceso de inclusión digital. El artículo culmina con unas sencillas orientaciones para la puesta en práctica de este tipo de acciones educativas, pretende ser una herramienta de utilidad para el trabajo de los educadores y educadoras, en el ámbito de la alfabetización en nuevas tecnologías.
\end{abstract}

Palabras claves: Alfabetización informática; brecha digital; inclusión digital.

\begin{abstract}
This article analyzes the digital divide in Spain from a bibliographic perspective, focusing on the digital literacy as a fundamental, pedagogical element. Educational interventions in new technologies have been the subject of analysis, both at an academic and institutional level. Many ideas and strategies have emerged from this analysis to carry out digital literacy projects. Thus, the present paper reflects the conceptualization and current situation of the digital divide, considering the interventions in digital literacy as an essential element in the process of digital inclusion. The article culminates with simple guidelines to implement this type of educational actions; these guidelines also intend to be a useful tool for the educators' work in the field of literacy in new technologies.
\end{abstract}

Keywords: Computer literacy; digital divide; digital inclusion.

\footnotetext{
${ }^{1}$ Máster oficial en Investigación e Innovación en Contextos Educativos por la Universidad de Cantabria y Diplomado en Educación Social por la Universidad del País Vasco. En mi trayectoria profesional, destaca el trabajo en diversas entidades de índole social, en el desempeño de tareas socio-educativas con diferentes colectivos. Por otro lado, colaboro como revisor en Revista Educare, donde publiqué en 2015 el artículo titulado "Derecho a la investigación y nuevas formas de difusión del conocimiento científico: El Open Access".
} 
doi: http://dx.doi.org/10.15359/ree.21-2.9

URL: http://www.una.ac.cr/educare

CORREO: educare@una.cr

La brecha digital (digital divide) se entendía, en su origen, como la mera existencia de un grupo excluido de la sociedad de la información, debido a la falta de posibilidades para acceder a los recursos tecnológicos. Posteriormente, esta categorización, comienza a adquirir complejidad, pues existen múltiples motivos por los que se produce la desigualdad digital (Castaño, 2008). Es decir, tenemos en cuenta que se producen desigualdades para acceder a las TIC, ya no solamente por una incapacidad material o de infraestructuras, sino que los factores por los que existen "personas excluidas digitales" son muy diversos.

Wessels (2010, en Ragnedda y Muschert, 2013), establece una serie de motivos por los que se produce la desigualdad digital. Así se observan:

- Aspectos socio-culturales como la etnia, edad, nivel académico y nivel económico.

- Las diferencias entre las infraestructuras de diferentes zonas del mundo. Alude a una desigualdad producida debido a las diferencias económicas globales.

- Escaso capital cultural para utilizar recursos tecnológicos, o falta de habilidades o conocimientos digitales.

En esta línea, Varela (2015) expone varios tipos de brecha digital (BD).

BD por cuestión de género: En España existe un nivel inferior en formación digital de las mujeres respecto a los hombres. Si bien, esta diferencia se ha ido disminuyendo en los últimos años$^{2}$, todavía existe una desigualdad superior a la mayoría de los países de la UE.

BD por cuestión de edad: Observando la variable edad, existen diferencias significativas en el uso de las tecnologías de la información y la comunicación (TIC). Por lo tanto, la brecha digital por edad es palpable, aunque se destaca una tendencia a su futura desaparición. Cada vez más personas de mediana y avanzada edad utilizan recursos tecnológicos. Así, se ha pasado de un 3\% de personas entre 65 y 74 años que utilizaban internet en 2004, a un 26,2\% en 2014. Para una mejor comprensión de este tipo de brecha digital, se expone a continuación una categorización de las "generaciones tecnológicas", con sus correspondientes características (Castaño, Martín, Martínez, Martínez-Espada y Vázquez, 2009):

- Generación tecnológica 1: Son las personas más jóvenes (no más de 30 años), nacidas en un entorno tecnológico, han incorporado competencias tecnológicas desde edades tempranas. Se les conoce también por la denominación de "personas nativas digitales".

\footnotetext{
${ }^{2}$ En el año 2005, existía una diferencia del 9\% en el uso de internet entre mujeres y hombres. En 2014, se aprecia un 3,4\% más de hombres que usan internet. La diferencia ha disminuido, no obstante, España sigue siendo uno de los países de la UE con mayor desigualdad de género en el uso de las TIC.
} 
- Generación tecnológica 2: Abarca un grueso de población, de mediana edad, marcadamente heterogénea, cuya inmersión TIC es intermedia. En este proceso han tenido influencia, especialmente, la formación recibida y el ámbito laboral.

- Generación tecnológica 3: Personas de mediana y avanzada edad, cuyo contacto con las nuevas tecnologías (NNTT) ha estado marcado por el desempeño de profesiones de alta cualificación, o bien, por las acciones formativas específicas en materia TIC.

BD de índole funcional: Referido a las personas con algún tipo de discapacidad, destacamos la existencia de una "barrera digital". Una dificultad de acceso a los recursos tecnológicos que genera un obstáculo más en la vida de personas con diversidad funcional. En este sentido, España se encuentra a la cola de la inclusión digital en personas con discapacidad. Varela (2015) destaca aquí que esta mayor dificultad de acceso a las TIC, por parte de la persona con discapacidad, anula su posibilidad de beneficiarse de las NNTT, y de los aspectos positivos que estas pueden aportarles.

BD económica: Se trata de la relación existente entre la renta que posee la ciudadanía y su acceso a recursos tecnológicos. Por lo tanto, esta variable depende de la capacidad monetaria de la persona. El 74\% de los hogares españoles que no dispone de internet se debe a motivos económicos (INE, 2013 en Varela, 2015).

BD geográfica: Las infraestructuras de las que dispone cada zona, dentro del Estado español, hace que existan diferencias significativas en el acceso a internet, dependiendo de la riqueza de cada comunidad autónoma. Así, mientras en Madrid, un 82,9\% de los hogares disponían de banda ancha, en Extremadura, el porcentaje es del 62,2\%.

BD urbana-rural:Se refiere a la brecha digital causada por el asilamiento, especial orografía o muy baja densidad de población. En este aspecto, las estadísticas revelan que España sobresale entre los países europeos, con un porcentaje de un 94,3\% de cobertura rural de banda ancha, cuando la media europea se sitúa en un $89,8 \%$. No obstante, sí se aprecian diferencias internas, entre localidades rurales y urbanas. Así, el porcentaje de hogares que no dispone de banda ancha en localidades de menos de 10.000 habitantes triplica al de las capitales de provincia.

BD laboral: La situación laboral es un elemento determinante en el uso de NNTT. Mientras el $15,13 \%$ de personal no utiliza internet, existe un porcentaje de $27,17 \%$ de personas desempleadas y un 35,68 de inactivas que no lo utilizan (INE, 2011 en Varela, 2015). Así mismo, en este tipo de brecha digital existe una fuerte contradicción, al disponer de internet casi la totalidad de las empresas españolas (95,3\%, por encima de la media UE: $90,0 \%$ ), aunque tan solo un $27 \%$ de su personal utiliza internet regularmente en el trabajo (Eurostat, 2014 en Varela, 2015).

BD por nivel formativo: Si bien, ligeramente por encima de la media europea, España no destaca por un alto grado de población formada en NNTT. Así, tan solo un $47,24 \%$ de su 
doi: http://dx.doi.org/10.15359/ree.21-2.9

URL: http://www.una.ac.cr/educare

CORREO: educare@una.cr

población posee un dominio medio o alto de internet. En esta falta de nivel formativo, se enmarca el fenómeno del "analfabetismo digital", eje principal en este escrito.

Para disminuir la brecha digital formativa o analfabetismo digital, encontramos las intervenciones educativas de "alfabetización digital" (digital literacy). Esta se define, siguiendo a Casado (2006, p. 52) como:

El proceso de adquisición de los conocimientos necesarios para conocer y utilizar adecuadamente las infotecnologías y poder responder críticamente a los estímulos y exigencias de un entorno informacional, cada vez más complejo, con variedad y multiplicidad de fuentes, medios de comunicación y servicios.

La alfabetización digital se ha desarrollado desde dos grandes paradigmas. Uno que defiende la necesidad de adquirir competencias digitales, para finalidades relacionadas con la competitividad y el crecimiento económico y, otra, desde la que se elaborarán las propuestas de intervención, que entiende la alfabetización digital como derecho de la ciudadanía, y para disminuir las desigualdades y exclusión social (e-inclusión) (Area, Gutiérrez y Vidal, 2012).

Pero, ¿cuáles son las causas del analfabetismo digital?

En la línea de Marín y González-Piñal (2011), se destacan tres motivos por los que se produce la desigualdad digital:

Analfabetismo digital por falta de acceso a la tecnología: Dificultad de acceso a las TIC, por circunstancias socioeconómicas o políticas (ej.: falta de recursos económicos, infraestructuras, determinados regímenes políticos censores, etc.)

Analfabetismo digital generacional: Suele manifestarse en personas de mediana y avanzada edad y se caracteriza por la negativa de estas a introducir las NNTT en su vida. Esta es una de las principales barreras que se encuentra en los procesos de alfabetización digital. Moreno (2008) apoya esta idea, al afirmar que existe una falta de concienciación de la importancia que supone la formación en competencias digitales, tanto entre la administración pública como en la ciudadanía en general. Lavilla (2006), por su parte, aporta el alarmante dato de la existencia de un $71 \%$ de ciudadanía española que considera "no necesario" acceder a internet.

Analfabetismo digital por analfabetismo tradicional: Se deriva del analfabetismo clásico. Un nivel académico-cultural excesivamente bajo, produce una gran dificultad para el manejo de las NNTT.

Por lo tanto, la esencia de la brecha digital radica en el estudio por comprender aquellos privilegios que otorga el uso de las NNTT a la persona, como búsqueda de empleo, acceso al 
conocimiento, defensa de derechos e intereses sociales, etc. Así, se viene hablando de "ventajas competitivas", término que hace referencia a "todo tipo de información, conocimiento y oportunidades que permitan a un individuo posicionarse en mejor situación para lograr optar a bienes competitivos o escasos" (Robles, Molina y De Marco, 2012, p. 797).

Además de las ya mencionadas ventajas que brindan las TIC a sus individuos usuarios, se destacan unas fuertes limitaciones que afectan a las personas que (por diversos motivos) no las manejan. En esta tesitura, cabe preguntarnos si, además de una limitación en el acceso a internet, con lo que ello conlleva, existe una mayor potenciación de los contenidos, valores e intereses por quienes sí utilizan las TIC.

Esta idea se relaciona con la de "ciudadanía digital", que Mossberger, Tolbert y McNeal (2008, en Robles et al., 2012) conceptualizan como la posibilidad de obtener algún tipo de beneficio social, político o económico de los recursos digitales, además de reseñar la dificultad que algunas personas tienen para ello, lo que dificultaría la creación (por lo menos equitativa) de dicha ciudadanía digital.

Es decir, tenemos en cuenta que no solamente se produce una dificultad para acceder a los contenidos de internet, por parte de quienes quedan al margen del mundo digital, sino que también existe una fuerte tendencia al predominio, en la construcción de la "ciudadanía digital", de los intereses de quienes manejan las NNTT.

En este sentido, se ha venido hablando de una "intersubjetividad digital". En pedagogía, denominamos intersubjetividad al principio de que las personas más capaces guían la acción de las menos capaces (Marín y González-Piñal, 2011). Por ejemplo, en la escuela, es el maestro o maestra quien guía el aprendizaje del alumnado. No obstante, esta concepción se invierte, dando lugar al concepto de intersubjetividad digital, en la que es el niño, niña o joven quien, marcado por su "herencia digital" (se recuerda la denominación de "nativos digitales"), maneja los procesos tecnológicos. No es raro, observar como personas adultas dejan en manos de jóvenes este tipo de tareas.

No cabe duda de la gran importancia que adquiere la alfabetización digital. Las intervenciones educativas de esta índole son, por lo tanto, necesarias para promover la inclusión digital y, en consecuencia, la igualdad de oportunidades en la ciudadanía.

Siguiendo a Martínez y Bermúdez (2012), señalamos las tres dimensiones que abarca la inclusión digital. Una de alfabetización digital (aprender a manejar los recursos tecnológicos), otra dimensión que hace referencia a la dotación y el mantenimiento de las infraestructuras (necesidad de contar con servicios de calidad para poder acceder al universo digital), así como la difusión "amplia y gratuita" del conocimiento (software libre, acceso a los recursos digitales etc.). En este último aspecto, añaden, las administraciones públicas tienen un papel de gran relevancia. 
doi: http://dx.doi.org/10.15359/ree.21-2.9

URL: http://www.una.ac.cr/educare

CORREO: educare@una.cr

A nivel institucional, se destaca la Agenda digital para España (Gobierno de España, Ministerio de Industria, Energía y Turismo, 2013), cuyo plan para la inclusión digital y empleabilidad cita como ejes en esta estrategia: mejorar la accesibilidad a las NNTT; avanzar en la alfabetización digital; reducir la desigualdad por cuestión de género y mejorar la empleabilidad a través de las TIC.

Los dos ejes que se persiguen en el plano de la alfabetización digital son los siguientes. Por un lado, el plan destaca la formación en materia TIC, especialmente, dirigido a los colectivos más desfavorecidos. Por otro lado, promueve las intervenciones formativas, dirigidas a aquellos colectivos con escaso contacto con las NNTT (personas mayores...).

Por su parte, la OCDE (2006, en Castaño, 2008) distingue tres tipos de usuarios o usuarias, en función de su grado de manejo de las NNTT:

Especialistas TIC: Las TIC son un eje fundamental en su empleo. Este tipo de usuarios pueden "crear y mantener" sistemas informáticos.

Personas usuarias avanzadas: Sin ser la informática el principal cometido en su trabajo, tienen conocimientos tecnológicos específicos, aplicados a su sector profesional.

Personas usuarias básicas: Referidas a aquellas personas alfabetizadas digitalmente que pueden utilizar, de forma competente, herramientas TIC, genéricas, para el desempeño de su actividad laboral y propone como competencias digitales necesarias, el manejo de: Word; Excel; PowerPoint y Outlook.

Hasta aquí, tenemos claros los conceptos e ideas claves para comenzar a teorizar acerca de las intervenciones educativas en materia de alfabetización en NNTT. Seguidamente, se exponen algunas orientaciones para tener una idea de los ámbitos que ha de abarcar un proyecto de alfabetización digital. A continuación, observamos la categorización elaborada por Shapiro y Hughes (1996, en Bawden, 2002).

- Alfabetización en herramientas. Manejo de los componentes informáticos: hardware y software.

- Alfabetización en recursos. Conocimiento de las diferentes formas de acceder a la red.

- Alfabetización socioestructural. Nociones sobre la producción de la información y su enmarque social.

- Alfabetización investigadora. Utilizar las TIC con finalidades académicas.

- Alfabetización en tecnologías incipientes. Abarca la comprensión de la innovación tecnológica y la capacidad para adaptarse a los cambios y tomar decisiones adecuadas en materia de NNTT.

- Alfabetización crítica. Evaluar con pensamiento crítico los beneficios y costes de las TIC. 
Si bien conocemos, a modo orientativo, las bases en las que ha de fundamentarse una intervención pedagógica en materia de alfabetización digital, debemos tener en cuenta también las metas presentes en el diseño de la intervención.

Las finalidades que han de perseguir este tipo de proyectos o, dicho de otro modo, lo que una persona debe ser capaz de "saber hacer" para considerarse digitalmente alfabetizada, se han clasificado del siguiente modo (Bruce, 1994 en Badwen, 2002):

- Adquiere competencias digitales de manera autónoma.

- Utiliza los procesos de información y la variedad de tecnologías existentes.

- Ha interiorizado los valores en los que se fundamentan las TIC.

- Tiene conocimientos sólidos sobre el mundo digital.

- Tiene pensamiento crítico en materia TIC.

- Interactúa con el mundo de las NNTT.

Una versión más específica del tipo de competencias que ha de adquirir el beneficiario o beneficiaria de estas intervenciones educativas es la que aporta Moreno (2008): Saber usar un navegador/buscador; gestionar el correo electrónico; saber utilizar los componentes ofimáticos (procesadores de texto, presentaciones, hoja de cálculo, etc.) y manejar componentes externos (cámara fotográfica, pen drive, etc.).

Así mismo, se ha venido hablando de "soltura" (fluency), término que hace referencia a la necesidad, no solamente de tener conocimientos y habilidades informáticas, sino habilidades de búsqueda, clasificación y presentación de la información, lo que se relaciona, en gran medida, con el nivel cultural de la persona (Castaño, 2008). En este sentido, Travieso y Planella (2008) exponen la necesidad de fomentar la inclusión digital, promoviendo el pensamiento crítico y la actitud activa, para trascender la mera adquisición de competencias digitales.

Seguidamente, se procede a mostrar algunas orientaciones para el diseño de proyectos educativos de alfabetización digital.

\section{Conclusiones}

Si bien son muy variados los análisis realizados, desde la perspectiva teórica, sobre la brecha digital y la alfabetización digital, se considera necesaria una mayor especificidad, por parte del discurso experto, sobre las pautas a seguir para poner en práctica acciones educativas de alfabetización en NN.TT. En este sentido, se plantean a continuación una serie de reflexiones, 
doi: http://dx.doi.org/10.15359/ree.21-2.9

URL: http://www.una.ac.cr/educare

CORREO: educare@una.cr

que se desprenden de la revisión bibliográfica y que pretenden suponer una aportación a la praxis de los educadores y educadoras en materia de nuevas tecnologías.

En este sentido, se considera necesario adaptar al máximo la intervención a las necesidades de la persona beneficiaria, atendiendo sus intereses. Se han de definir bien los objetivos necesarios para la adquisición de competencias digitales y no caer en el error de sobrecargar con otros contenidos (aunque puedan ser interesantes) alejados del objetivo inicial. Esta es una variable relacionada directamente con la formación específica de competencias digitales.

Para la elaboración de objetivos, tenemos en cuenta lo expuesto anteriormente y que podría resumirse, recordando a Bruce (1994, en Bawden, 2002):

Que el sujeto usuario pueda construir un aprendizaje autónomo en el mundo digital; que pueda utilizar la gran variedad de TIC existentes, que adquiera conocimientos óptimos (además del ordenador, teléfonos móviles, tabletas, etc.); que conozca en qué se fundamentan las NNTT y sea consciente de su importancia en la sociedad actual; y que desarrolle un pensamiento crítico en materia digital.

Además, quelas intervenciones educativas deben atender los intereses delapersonausuaria, para lo cual es conveniente buscar aplicaciones prácticas de los conocimientos informáticos. Es decir, dotar de una utilidad cotidiana a los conocimientos que se adquieren en la clase. Esta aplicación práctica es un elemento de gran importancia en los procesos de alfabetización digital, ya que favorece la inclusión digital de la persona beneficiaria como sujeto crítico y activo.

No olvidamos la necesidad de adecuar el nivel. Esto es, hemos de conocer el nivel de informática que tiene el grupo para poder, en función de sus intereses y su nivel TIC, ofrecerle contenidos adecuados, siguiendo la norma general de proponer "metas difíciles, pero alcanzables" ${ }^{\prime \prime}$.

Por otro lado, debemos elaborar el material desechando un gran abanico de terminología técnica que podría generar rechazo en la persona usuaria, así como hacer que el proyecto rompa con los estereotipos existentes en materia $\mathrm{TIC}$, los cuales, tenemos en cuenta que no son naturales sino construidos (Casado y Díez, 2006). Esto es, la persona alejada del mundo digital ha construido una serie de ideas estereotipadas, que los educadores y educadoras debemos cambiar, para promover el proceso de aprendizaje tecnológico.

En suma, un elemento clave que se ha de tener en cuenta es la población destinataria (individuos desempleados, personas mayores, colectivos en riesgo de exclusión, etc.) y detectar sus intereses por la informática, sus necesidades y nivel formativo, para lo que se recuerda, existen múltiples métodos investigativos (encuesta, entrevista, etc.) de gran utilidad en este proceso.

${ }^{3}$ Se recuerda la teoría de Vygotski: zona de desarrollo próximo. 
Por último, se reseña la importancia del formador o formadora que ejecuta el proyecto. Un equipo profesional con competencias digitales y sólida formación pedagógica, será un elemento clave en el éxito de este tipo de intervenciones.

\section{Referencias}

Area, M., Gutiérrez, A. y Vidal, F. (2012). Alfabetización digital y competencias informacionales. Fundación Telefónica. Barcelona: Ariel. Recuperado de https://ddv.stic.ull.es/users/ manarea/public/libro \%20Alfabetizacion digital.pdf

Bawden, D. (2002). Revisión de los conceptos de alfabetización informacional y alfabetización digital. Anales de Documentación. 5, 361-408. Recuperado de http://revistas.um.es/ analesdoc/article/view/2261

Casado, R. (2006). Alfabetización digital: ¿Qué es y cómo debemos entenderla? En R. Casado (Coord.), Claves de la alfabetización digital (pp. 51-55). Barcelona: Ariel.

Casado, R. y Díez, E. (2006). 13 claves de la alfabetización digital. En R. Casado (Coord.), Claves de la alfabetización digital (pp. 203-214). Barcelona: Ariel.

Castaño, C. (2008). La segunda brecha digital. Madrid: Cátedra.

Castaño, C., Martín, J., Martínez, J. L., Martínez-Espada, E. y Vázquez, S. (2009). Las generaciones tecnológicas en la segunda brecha digital. Un análisis de su incidencia sobre la incorporación efectiva de las mujeres a internet. Madrid: Observatorio e-igualdad.

Gobierno de España, Ministerio de Industria, Energía y Turismo. (2013). Agenda digital para España. España: Autor. Recuperado de http://www.agendadigital.gob.es/agenda-digital/ recursos/Recursos/1.\%20Versi\%C3\%B3n\%20definitiva/Agenda Digital para Espana.pdf

Lavilla, F. (2006). España Avanz@. En R. Casado (Coord.), Claves de la alfabetización digital (35-40). Barcelona: Ariel.

Marín, I. y González-Piñal, R. (2011). Relaciones sociales en la sociedad de la información. Prisma Social, 6, 119-137. Recuperado de http://dialnet.unirioja.es/servlet/ articulo?codigo $=3686079$

Martínez, P. C. y Bermúdez, M. T. (2012). La brecha digital. Una nueva línea de ruptura para la educación social. RES. Educación Social, 14, 1-9. Recuperado de http://www.eduso.net/res/ pdf/14/brecha res $14 . p d f$ 
doi: http://dx.doi.org/10.15359/ree.21-2.9

URL: http://www.una.ac.cr/educare

CORREO: educare@una.cr

Moreno, M. D. (2008). Alfabetización digital. El pleno dominio del lápiz y el ratón. Comunicar. Revista Científica de Comunicación y Educación, 15(30), 137-146. Recuperado de http:// www.revistacomunicar.com/verpdf.php?numero $=30$ \&articulo $=30-2008-22$

Ragnedda, M. y Muschert, G. W. (2013). The digital divide. The internet and social inequality in internacional perspective. New York: Routledge.

Robles, J. M., Molina, Ó. y De Marco, S. (2012). Participación política digital y brecha digital política en España. Un estudio de las desigualdades digitales. ARBOR Ciencia, pensamiento y cultura, 188(756), 795-810. doi: 10.3989/arbor.2012.756n401

Travieso, J. L. y Planella, J. (2008). La alfabetización digital como factor de inclusión social: Una mirada crítica. Uocpapers, 6, 1-9. Recuperado de http://www.uoc.edu/uocpapers/6/dt/ esp/travieso planella.pdf

Varela, J. (2015). La brecha digital en España. Estudio sobre la desigualdad postergada. Madrid: Comisión ejecutiva confederal de UGT. Secretaría de participación sindical e institucional. Recuperado de http://www.ugt.es/Publicaciones/BRECHADIGITAL WEB.pdf 